\title{
Traffic aware data collection MAC protocol for wireless sensor networks.
}

\begin{abstract}
In this paper, we propose a new medium access control (MAC) protocol for wireless sensor networks for periodic data collection. The proposed MAC scheme is specifically designed for wireless sensor networks which have periodic traffic with different sampling rates. In our protocol design, sink determines the time schedule for all other nodes in the network. We discuss the design of traffic aware MAC protocol and provide a comparison with the similar MAC protocols through simulation. Under different traffic generation rate, our protocol outperforms the previous one in terms of energy consumption and packet delay. Simulation results show that our protocol demonstrates up to $35 \%$ better performance than that of most recent protocol which is proposed for this kind of application, in respect of energy consumption. Comparative analysis and simulation results show that our design considerably gives a good compromise between energy efficiency and packet latency.
\end{abstract}

Keyword: Medium access control; Wireless sensor network; Periodic data collection; Traffic awareness. 